\title{
Motion Tracking of a Human Subject in Healthcare Applications Using Compact Ultra Wideband Antennas
}

\author{
Richa Bharadwaj, S. Swaisaenyakorn*, Clive Parini, J. Batchelor*, and Akram Alomainy \\ Antenna \& Electromagnetics Research Group, School of Electronics and Computer Science Engineering \\ Queen Mary University of London, London, UK \\ *School of Engineering and Digital Arts, University of Kent, Canterbury, UK \\ \{r.bharadwaj; a.alomainy\}@qmul.ac.uk
}

\begin{abstract}
This paper presents a study of 3D localisation of compact body-worn sensors using Ultra Wideband technology. Nine different sensor locations on the upper body of the human subject are considered. 3D localisation of the body worn sensors is achieved using time of arrival and peak detection techniques. The average 3D positioning accuracy achieved is in the range of 1 to $3 \mathrm{~cm}$ which are comparable with the complex optical motion capture system that is used as reference. Results also show that accuracy in estimation of location of the sensor depends on the position it is placed on the human body, configuration of the base station and the propagation phenomenon in the indoor environment in which localisation is taking place. The high $\mathrm{cm}$ accuracy obtained makes UWB technology suitable for various indoor applications in field of healthcare, sports, industry and day to day life.
\end{abstract}

Keywords- Body worn sensors, UWB, Time of Arrival, Localisation

\section{INTRODUCTION}

Ultra-wideband (UWB) has become a promising wireless technology in commercial and personal applications such as next generation short range and high data rate wireless communications, high resolution imaging and for accurate positioning [1]. Monitoring and classification of human activity using simple and compact body-worn sensors is emerging as an important research area in wireless communications. For indoor 3D human tracking, there are many technological hurdles to overcome due to the complexity of indoor environments [2,3]. The influence of obstacles like walls, equipments, furniture, presence of people etc. leads to fading and multipath issues, hence affecting the wireless radio propagation channel. Due to extremely narrow pulses, Impulse-Radio Ultra Wideband systems have emerged as a promising solution for high resolution indoor positioning and ranging applications. UWB meets the key requirements of localisation in terms of low cost, high data rate, easy implementation and low energy consumption [2-5]. UWB also has fewer effects on humans because of its low transmission power making it suitable for wearable sensors tracking and position estimation.
Commercial UWB localisation systems such as Ubisense, Multispectral Solutions Inc, have an accuracy of $10-15 \mathrm{~cm}$ [4-6]. Higher accuracy has been reported in the literature for indoor UWB positioning systems [2-6]. For example, Low et al. achieved centimetre-range accuracy in a 1-D short-range indoor LOS environment utilizing UWB pulse signals. Zetik et al. reported sub-millimetre 1-D accuracy, but with only extremely short displacements, while accuracy decreased to $1.5 \mathrm{~cm}$ for 2-D localization over a $2 \times 2 \mathrm{~m}$ area [4]. An accuracy of centimetre range is being achieved using impulse based UWB systems and sub -millimetre range accuracy is possible using carrier based UWB systems as proposed in literature [6].

In this work, UWB human body localisation is studied using wearable compact sensors placed on the nine different locations of the upper body in an indoor environment using analytical and numerical techniques. An optical motion capture system is used as reference for comparing the results obtained through UWB localisation technique. The objective of the work is to achieve accurate $3 \mathrm{D}$ localisation and directional information of the human body using data fusion time of arrival and peak detection techniques.

\section{MEASUREMENT SET UP}

Tapered co-planar waveguide fed UWB antennas (TSA) (Fig.1 [7]) were used as transmitters placed on the body and also as receivers. The TSA is a compact and cost effective antenna (size of $27 \mathrm{~mm} \times 16 \mathrm{~mm}$ ) with an excellent impedance matching for the 3 to $10 \mathrm{GHz}$ frequency range. It has a return loss better than $10 \mathrm{~dB}$ and good radiation performance in the UWB range with relatively constant gain across the whole frequency band [7].

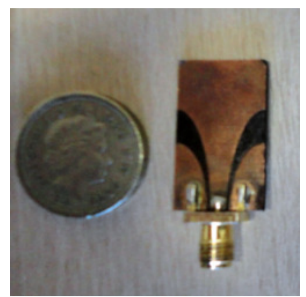

Figure 1. Tapered slot co-planar ultra wideband antenna. 
Measurements were performed at the motion capture studio at University of Kent, UK [8]. A real human test subject (1.68 $\mathrm{m}$ tall and average male build) was chosen for localizing sensors on the body. Nine sensor locations were chosen with three at the joints of both the arms and three on the torso. The distance between the human body surface and the antenna was around $5 \mathrm{~mm}$. Frequency domain measurements were performed in the 3 to $10 \mathrm{GHz}$ band. A vector network analyzer (VNA) was used to capture $S_{21}$ parameters between the body worn sensor and base station antenna. The antennas were mounted on plastic frames with 3 markers each to allow estimation of position of antennas in 3D space through VICON motion capture system [8]. The system consists of 8 cameras giving high accuracy in range of 1 to $2 \mathrm{~cm}$. The motion capture system was used to compare with the UWB localisation results and also to obtain exact coordinates of the base stations. The subject was made to stand in the centre of the 1.8 by $1.8 \mathrm{~m}^{2}$ area. The four base stations are positioned near the vertices of the cuboid to obtain high accuracy positioning in three dimensions [2,9]. The antenna at BS1 is considered as zero co-ordinate $\left(x_{0}, y_{0}, z_{0}\right)$ which acts as reference to find the position of the target placed inside the volume of the cube.
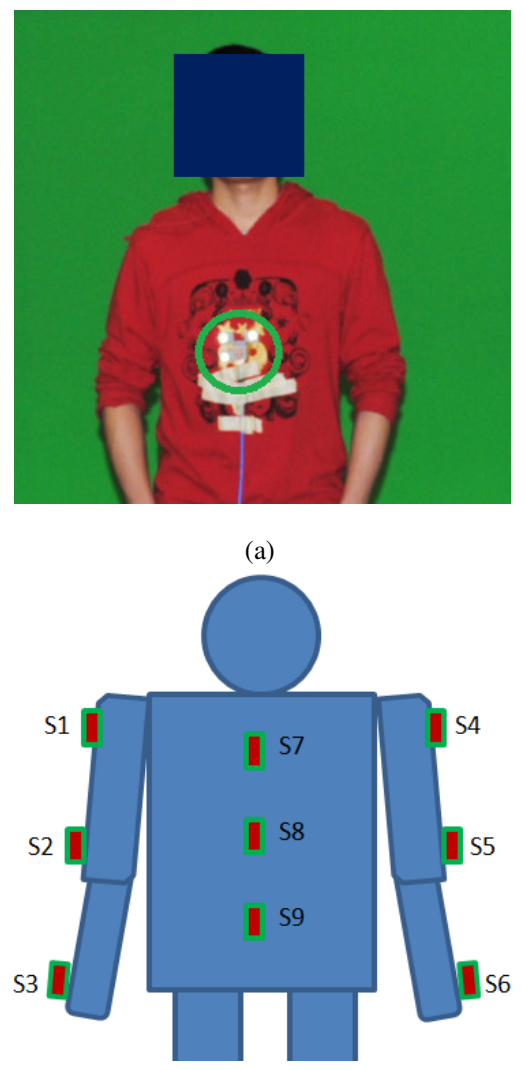

(b)

Figure 2. Human subject (a) standing in the centre of $1.8 \mathrm{X} 1.8 \mathrm{~m}^{2}$ area. The TSA antenna (body worn sensor) shown in green circle is placed on the centre of the chest. Schematic of the human subject (b) showing sensor locations on the upper body.

\section{LOCALISATION TECHNIQUES AND ALGORITHMS}

Time of Arrival positioning technique outperforms other techniques such as RSS-based, AOA-based positioning due to the fine time resolution of the UWB signal. It has been recognized that TOA technique can best deal with fine time resolution with the help of UWB technology [2, 9-11]. In this work a two-stage positioning technique (Fig. 3) is used in which certain parameters are extracted from the signals first, and then the position is estimated based on those signal parameters [5]. The accuracy in positioning can be enhanced by precisely finding the time of arrival (TOA) which depends on various parameters like bandwidth range, the width of the source pulse, type of source pulse transmitted, antenna efficiency and characteristics of the UWB communication system, etc [10].

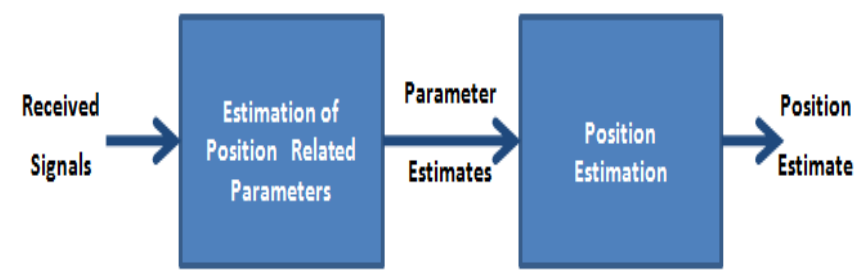

Figure 3. Two Step Positioning Technique. Step I: Estimation of position related parameters. Step 2: Position Estimation of the mobile target.

\section{A. Time of Arrival Estimation}

In the first step of a two-step positioning technique signal parameters, such as TOA, angle-of-arrival (AOA), and/or received signal strength (RSS), are estimated. The time of arrival between the mobile and base stations is estimated by considering channel impulse response (CIR) technique. $S_{21}$ parameters between transmitter and each receiver are measured on the vector network analyzer. An Inverse Fast Fourier Transform (IFFT) is then applied to obtain the impulse response of the measured channels (CIR). The channel impulse response $[9,10]$ is given by:

$$
h(\tau, t)=\sum_{k=1}^{K} a_{k}(t) \delta\left(\tau-\tau_{k}\right) e^{j \theta_{k}(t)}
$$

where $\delta$ is the Dirac delta function, $\mathrm{K}$ is the number of resolvable multipath components, $\tau_{k}$ are the delays of the multipath components, $a_{k}$ are the path amplitude values and $\theta_{k}$ are the path phase values.

The complex propagation phenomenon in the indoor environment gives rise to number of error sources that may degrade the accuracy of the Time of Arrival estimation between each BS and MS, such as thermal noise, multipath propagation, Direct Path (DP) blockage between Tx and Rx and DP excess delay [5, 9-10]. Line of Sight and Non Line of sight scenarios are observed with respect to different base station positions. For Line-of-sight situations (Fig. 4 (a), blue 
line), the TOA can be accurately estimated by the maximum peak detection technique. This is due to the large bandwidth of the UWB signal through which multipath components get usually resolved. The main challenge in UWB ranging is resolving dense multipath conditions and Non Line of sight (NLOS) situations which cause propagation delay and signal distortion. Fig. 4 (b) shows NLOS situation with high multipath present which induces positive biases in distance estimation leading to high error in time of arrival estimation. Threshold based algorithms [9-10] such as search back and leading edge detection techniques are used in this work in order to mitigate NLOS situations and improve time of arrival estimation accuracy.

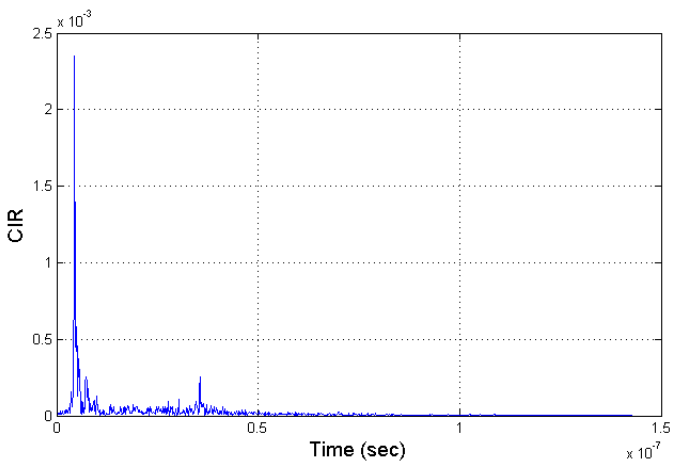

(a)

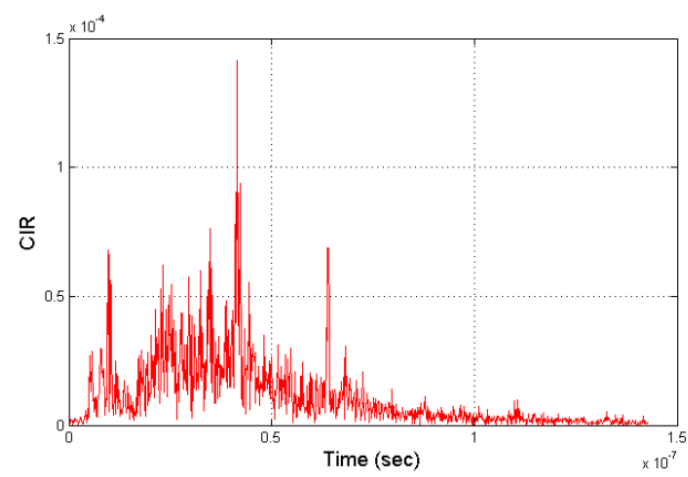

(b)

Figure 4. Body-worn sensor Channel Impulse Response: (a) Line of Sight and (b) Non Line of Sight Situations.

\section{B. Linear Least Square Algorithm}

In the second stage, the position of the mobile station is determined based on the signal parameters obtained in the first stage. Trilateration and triangulation are two most popular geometric approaches that are used to estimate the unknown position of the target node. In this work, time of arrival (TOA) data fusion method [11] is used to find the MS position which is based on combining estimates of the TOA of the MS signal that arrives at four different BSs. Let $\left(r_{1}, r_{2}, r_{3}, r_{4}\right)$ represent the range measurements obtained from the distance between MS and BS. The equations are solved jointly by using least square solution in order to estimate the position of the body worn sensor $\left(x_{m}, y_{m}, z_{m}\right)$ via trilateration:

$$
r_{i}^{2}=\left(x_{i}-x_{m}\right)^{2}+\left(y_{i}-y_{m}\right)^{2}+\left(z_{i}-z_{m}\right)^{2}
$$

where $i=1,2,3,4$

\section{LOCALISATION ACCURACY ANALYSIS}

Results for the localisation of the body worn sensors on the human subject are shown in Fig. 5. High accuracy in the range of 1 to $3 \mathrm{~cm}$ is achieved using time of arrival UWB localisation techniques. The presence of the human body causes interference and delay in transmission of the signal from the base stations to the sensor on the body, hence affecting the channel propagation characteristics and the overall localisation accuracy. As shown in Fig. 5 the torso region shows higher error which is due to the presence of human body which acts like an obstacle for signals to pass especially for the BS3 and BS4 which are facing the back of the human subject. The average localisation accuracy for directional azimuth and elevation angles with respect to BS 1 is approx. 1 to 2 degrees.

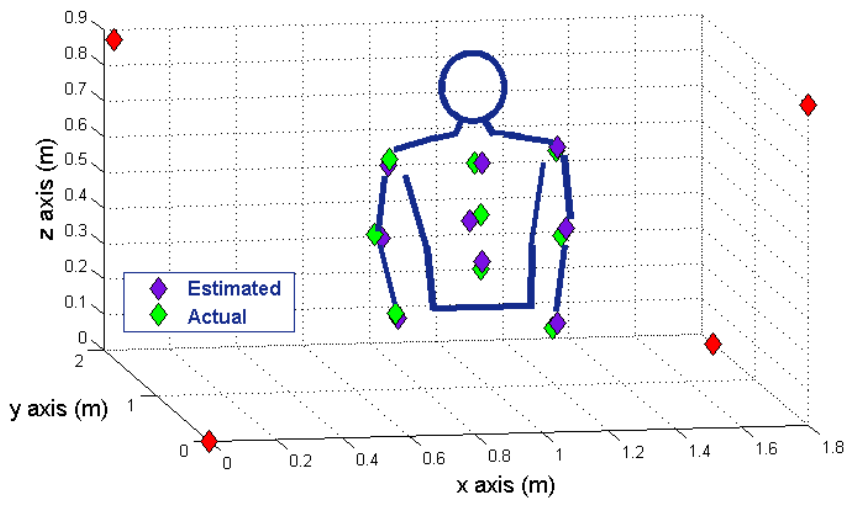

Figure 5. Comparison of actual and estimated positions of the body worn sensors placed on the upper body of the human subject. The markers in red represent the four Base Station positions.

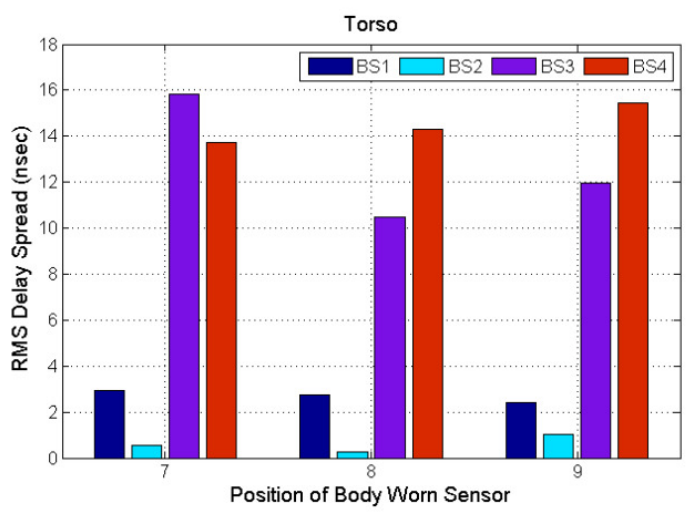

Figure 6. Average RMS delay spread obtained for the four Base Stations used to localize body worn sensors (Sensor placed near right elbow and centre of chest). 
The root mean square (RMS) delay spread is defined as the square root of the second central moment of the power delay profile [1,5] and also describes the time dispersion characteristics of the channel.The variation in RMS delay spread depends on the position of the wearable sensors, base station configuration and the indoor environment. Fig. 6 shows a comparison of the RMS delay spread (Torso region) for the four Base Stations with respect to sensor location. It can be observed that BS1 and BS 2 are in LOS situation as the BS's are facing the front of the human subject and hence show lower values of RMS delay spread. In general BS3 and BS4 show higher values of RMS delay spread as the sensors are facing the back of the human subject which gives an indication of high multipath, delay and more probability of NLOS situations. The RMS delay spread for the torso region is higher for the sensors BS3 and BS4 because this region acts like an obstruction for signal to transmit between the sensors and the BS's leading to high multipath propagation.

\section{CONCLUSION}

3D localisation has been obtained using compact and cost effective wearable Ultra Wideband antennas placed on the upper body of a human subject in an indoor environment. Time of Arrival data fusion, Channel Impulse Response technique and first peak detection algorithms are used to estimate the location of the unknown target. High 3D accuracy is obtained in centimetre range $(1 \mathrm{~cm}$ to $3 \mathrm{~cm})$ suitable for wearable sensor network applications such as tracking, patient monitoring, training of athletes and motion capture. The localisation accuracy achieved is comparable with the complex optical motion capture system. The work carried out gives an insight regarding the propagation phenomenon in presence of a human body in the localisation area and the accuracy achieved while localizing the sensors present on the human body.

\section{REFERENCES}

[1] Gezici, S.; Poor, H.V.; , "Position Estimation via Ultra-Wide-Band Signals," Proceedings of the IEEE, vol.97, no.2, pp.386-403, Feb. 2009.

[2] Bharadwaj, R.; Alomainy, A.; Parini, C., "Localisation of body-worn sensors applying Ultra Wideband technology," Antennas and Propagation (APCAP), 2012 IEEE Asia-Pacific Conference on, vol., no., pp.106,107, 27-29 Aug. 2012.

[3] D. Zhang, F. Xia, Z. Yang, L. Yao, W. Zhao, Localization technologies for indoor human tracking, in: Proceedings of the IEEE International Conference on Future Information Technology (FutureTech'10), Pusan, Korea, May 2010.

[4] R. Zetik, J. Sachs, R. Thomä, "UWB localization - active and passive approach," in Proceedings of the 21st IEEE IMTC, vol. 2, 2004, pp. 1005-1009.

[5] Z. Sahinoglu, S. Gezici, and I. Guvenc, Ultra-Wideband Positioning Systems: Theoretical Limits, Ranging Algorithms, and Protocols, Cambridge University Press, 2008.

[6] Mahfouz, M.R.; Cemin Zhang; Merkl, B.C.; Kuhn, M.J.; Fathy, A.E.; , "Investigation of High-Accuracy Indoor 3-D Positioning Using UWB Technology," Microwave Theory and Techniques, IEEE Transactions on , vol.56, no.6, pp.1316-1330, June 2008

[7] Alomainy, A.; Sani, A.; Rahman, A.; Santas, J.G.; Yang Hao; , "Transient Characteristics of Wearable Antennas and Radio Propagation Channels for Ultrawideband Body-Centric Wireless Communications," Antennas and Propagation, IEEE Transactiovol.57, no.4, pp.875-884, April 2009.

[8] Swaisaenyakorn, S.; Young, P.R.; Kelly, S.W.; Batchelor, J.C., "Comparison of 3D scanned human models for off-body communications using motion capture," Antennas and Propagation Conference (LAPC), 2012 Loughborough, vol., no., pp.1,4, 12-13 Nov. 2012

[9] Bharadwaj, R.; Parini, C.; Alomainy, A., "Ultra wideband-Based 3-D Localization Using Compact Base-Station Configurations," Antennas and Wireless Propagation Letters, IEEE, vol.13, no., pp.221,224, 2014.

[10] Alsindi, N.A.; Alavi, B.; Pahlavan, K., "Measurement and Modeling of Ultrawideband TOA-Based Ranging in Indoor Multipath Environments," Vehicular Technology, IEEE Transactions on, vol.58, no.3, pp.1046,1058, March 2009.

[11] Sayed, A.H.; Tarighat, A.; Khajehnouri, N.;, "Network-based wireless location: challenges faced in developing techniques for accurate wireless location information," Signal Processing Magazine, IEEE , vol.22, no.4, pp. 24- 40, July 2005. 